\title{
EDITORIAL
}

\section{VIRTUAL GROUPS TO ADDRESS THE HEALTH OF HOMEBOUND ADULTS DURING COVID-19: A BIOPSYCHOSOCIAL FRAMEWORK}

\author{
M. ZUBATSKY ${ }^{1}$

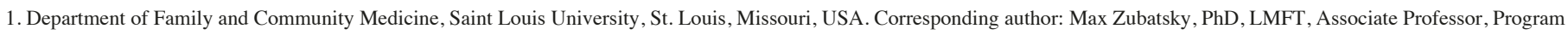
Director, Medical Family Therapy Program, School of Medicine, Saint Louis University, 3700 Lindell Boulevard, St. Louis, MO 63108, USA, Email: max.zubatsky@health.slu.edu

Key words: COVID-19 pandemic, dementia, fall risk, social isolation, telehealth.

The COVID-19 pandemic has presented a significant burden on older adults with ongoing health risks and conditions. Whether affected directly by the virus or staying homebound due to restrictions, the pandemic has affected both physical and mental capacities for this population (1). As a result, isolated older adults are at greater risk for developing depression, anxiety, stress-induced illness, suicidal ideation, substance use, and other underlying medical complications $(2,3)$. Lack of mobility and mental activity during the pandemic have also been heightened during the restrictions from COVID. One growing concern in the aging population both prior to and during the pandemic has been the social isolation of seniors who are separated from family, friends, and other social networks (4).

Social isolation has been a rising public health concern in the 65 and older age group across the world. Increased risks of mortality and long-term health risks have resulted from a lack of social connections and increased insecurity of making new contacts and friends (5). Individuals with cognitive impairment issues are now faced with a "double burden," as the pandemic has heightened their vulnerability due to both increased health risks from exposure and restricted access to care services due to increased lockdown measures. Caregivers and family members are now strained to find adequate resources and activities, especially for those with financial and other socioeconomic restrictions (6).

These health inequalities and disparities continue to exist for minority populations and those without access to curb isolation during COVID-19. Access to care providers and essential geriatric resources have been extremely limited for underserved populations in both rural and urban settings (7). Seniors in disadvantaged neighborhoods or residencies may also have limited financial or social means for technological devices to help connect with essential providers in their medical and social networks $(8,9)$. The pandemic has resulted in an immediate call to action for healthcare settings to help reach low socioeconomic and minority seniors through more innovative and collaborative means of communication (10).

As a response to the growing needs of adults requiring remote services and connections to improve their health, providers have been strategizing ways to connect virtually with a population often challenged with technology use (11). Primary Care Physicians, Geriatricians, and nurses have now adapted to video visits with the assistance of family and professional caregiver support. Home health workers have attempted to install tablets and other devices in residencies of older adults to help connect with providers, family, and friends (12). The recent modifications of online group and virtual connections have been quickly emerging as additional services offered by healthcare and community settings.

Several virtual adaptations for older adults have now been developed to address the immediate needs around health decline and social disconnection. Many of these online groups have had challenges adapting protocols and activities that often require in-person contact. Online groups often necessitate the participation of the primary caregiver or family member to help setup technology or connection of services. Attending to the holistic areas of older adult health including physical and nutritional areas $(13)$ and cognitive activity $(6,14)$ are essential to help maintain other health factors and functioning. We offer the following three virtual groups that have been gradually adapted virtually and address the biopsychosocial areas of older adults during the COVID-19 pandemic (figure 1).

\section{Figure 1}

Virtual Groups to Address Biospsychosocial Areas of Homebound Older Adults

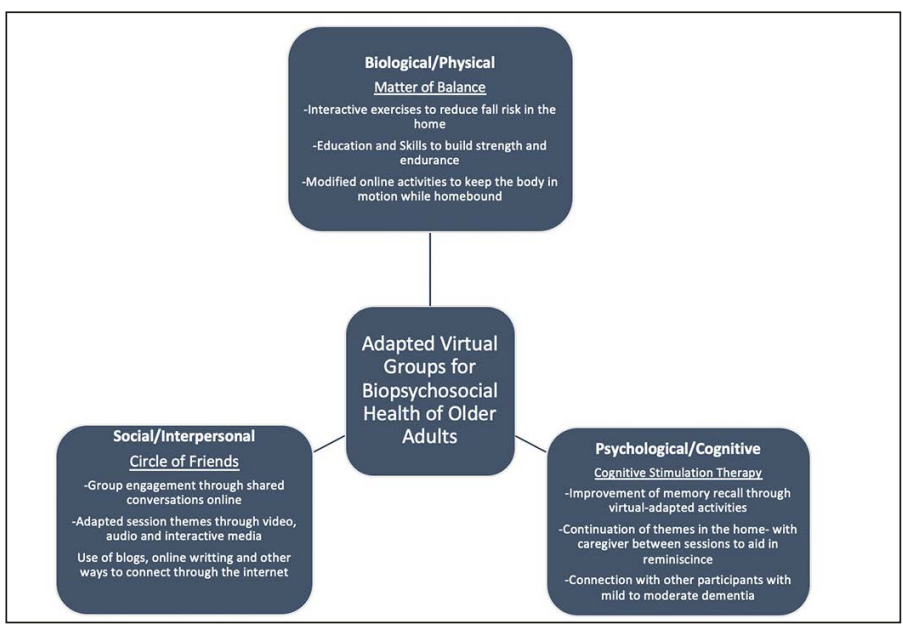

Published online January 8, 2021, http://10.1007/s12603-021-1584-8 
Matter of Balance (MOB)- Fall prevention and coordination has been an increasing concern for older adults during the homebound period of COVID-19. MOB is a program designed to help reduce the risk of falls and help adults gain skills to be more independent in their home environment. The protocol is an 8 -week intervention that emphasizes practical skills to prevent falls and increase activity levels. The group sets realistic goals to help avoid sarcopenia and atrophy of muscles. Virtual groups are starting to modify certain chair and standing activities that can be done in the comfort of one's home. Virtual rehabilitation interventions, computer assisted technologies, and virtual simulations $(15,16)$ can help participants mirror certain activity and exercises seen on the screen by facilitators. Individuals can utilize "sit-to-stand", chair, and other movement exercises to increased stability and prevent risks of falls in the home. These rehabilitation programs are especially needed in remote areas (17), where access to community activity groups is very limited.

Cognitive Stimulation Therapy (CST)- With very few nonpharmacological interventions that have shown effectiveness of those with cognitive deficits $(18,19)$, older adults with memory loss have struggled finding reminiscence groups and activities to help stimulate areas of the brain. CST is an evidenced-based intervention that uses reminiscence sessions to help aid recall of memory and improve mood of adults with mild to moderate dementia (20). The protocol is outlined to last for 14 sessions, twice per week over seven weeks. Activities are tailored to different skill levels of participants and help encourage thoughts and opinions rather than factual answers. Sample sessions cover themes such as food, money, childhood, word games, face recognition, and interactive games. Groups that have transitioned to a virtual platform during COVID have benefited both in the convenience of groups and the engagement of participants in stimulating areas of memory (21). Groups have now adapted additional activities and sessions that include virtual tours to cities and places, virtual reality videos, and interactive movements of faces, objects, and numbers on the screen.

Circle of Friends (CoF)- Developing more telehealth and virtual platforms will be critical for not just physical needs of older adults, but also social connection to reduce risks of loneliness and withdrawal from other community networks. Prior to COVID-19, rates of loneliness and major depression in the aging population was becoming a global epidemic, where non-pharmacological interventions were needed to expand connections and friendships of older adults. COF was an intervention developed to help alleviate loneliness and connect community members together as a participant-driven group (22). Groups meet once per week for 12 weeks, where facilitators check-in with participants about ways they have been coping with loneliness, while offering suggested activities to explore themes around physical activity, arts and hobbies, and therapeutic writing. The long-term goals of the group are to have participants lead discussions around loneliness and facilitate ideas to increase connection and community amongst its members (23). Online groups have used virtual connection to utilize screen sharing of video, music, interactive tours, and other forms of media to help groups members share common experiences online. Members are even bringing their own music, poems, and writing samples to share with members through the internet (24).

These suggested group interventions that have been modified virtually is not an inclusive list of resources for older adult health during COVID-19. Agencies and medical settings should continue to develop ways for providers and community partners to establish groups to connect adults through the remainder of the pandemic and beyond (25). Providers in the aging field are beginning to recognize the need for more "push" interventions in reaching vulnerable seniors who otherwise cannot leave their residence during the pandemic. The aftermath of older adults remaining isolated even after vaccine availability for COVID-19 will necessitate the continual development of virtual connection for older adults.

Conflict of interest: The author declares there are no conflicts.

Funding: This project is supported by the Health Resources and Services Administration (HRSA) of the U.S. Department of Health and Human Services (HHS) under grant number U1QHP28716, Geriatrics Workforce Enhancement Program, for $\$ 750,000$. This information or content and conclusions are those of the author and should not be construed as the official position or policy of, nor should any endorsements be inferred by HRSA, HHS, or the U.S. Government.

\section{References}

1. Shahid, Zainab, et al. COVID-19 and older adults: what we know. J of the Am Ger Soc. 2020; 68: 926-929. doi: 10.1111/jgs.16472

2. Garnier-Crussard, Antoine, et al. Novel Coronavirus (COVID-19) Epidemic: What Are the Risks for Older Patients?. J of the Am Ger Soc. 2020;68:939-940. doi: 10.1111 /jgs. 16407

3. Hämmig, O. Health risks associated with social isolation in general and in young, middle and old age. PLoS One. 2019;14: e0219663. doi: 10.1371/journal pone. 0219663

4. Vahia, IV., et al. COVID-19, mental health and aging: A need for new knowledge to bridge science and service. Am J of Ger Psych. 2020; 28:695-697. doi: 10.1016/j. jagp.2020.03.007

5. Cacioppo JT, Cacioppo S. The growing problem of loneliness. The Lancet. 2018; 391:426. doi: 10.1016/S0140-6736(18)30142-9

6. Brown EE, Kumar S, Rajji TK, Pollock BG, Mulsant BH. Anticipating and Mitigating the Impact of COVID-19 Pandemic on Alzheimer's Disease and Related Dementias. Am J of Ger Psych. 2020; 28:712-721. doi: 10.1016/j.jagp.2020.04.010

7. Cyr ME et al. Access to specialty healthcare in urban versus rural US populations: a systematic literature review. BMC health services research. 2019;19:1-17. doi 10.1186/s12913-019-4815-5

8. Jaffe DH et al. Health Inequalities in the Use of Telehealth in the United States in the Lens of COVID-19. Pop Health Man. 2020; 23:368-377. doi: 10.1089/pop.2020.0186

9. Schifeling $\mathrm{C}$ et al. Disparities in Video and Telephone Visits Among Older Adults During the COVID-19 Pandemic: Cross-Sectional Analysis JMIR Aging 2020;3:e23176. doi:10.2196/23176

10. Zhai Y. A call for addressing barriers to telemedicine: health disparities during the COVID-19 pandemic. Psychotherapy and Psychosomatics. 2020:1. doi: $10.1159 / 000509000$

11. Bianchetti A, Bellelli G, Guerini F, Marengoni A, Padovani A, Rozzini, R, \& Trabucchi M. Improving the care of older patients during the COVID-19 pandemic. Aging Clin and Exp Res. 2020;32:1883-1888. doi:10.1007/s40520-020-01641-w

12. Nicol GE, Piccirillo JF, Mulsant BH, Lenze EJ. Action at a Distance: Geriatric Research during a Pandemic. J Am Geriatr Soc. 2020;68:922-925. doi: 10.1111/ jgs. 16443

13. Solfrizzi $\mathrm{V}$ et al. Nutritional intervention as a preventive approach for cognitiverelated outcomes in cognitively healthy older adults: a systematic review. J of Alz Disease. 2018;64: S229-S254. doi: 10.3233/JAD-179940

14. Azarpazhooh MR et al. Correlations between COVID-19 and burden of dementia: 


\section{THE JOURNAL OF NUTRITION, HEALTH \& AGING}

An ecological study and review of literature. J of the Neuro Sciences. 2020;416: 117013. doi: $10.1016 /$ j.jns.2020.117013

15. Shubert TE. Evidence-based exercise prescription for balance and falls prevention a current review of the literature. J of Ger Phys Ther. 2011;34: 100-108. doi: 10.1519/JPT.0b013e31822938ac

16. Donath L, Roland R, Oliver F. Effects of virtual reality training (exergaming) compared to alternative exercise training and passive control on standing balance and functional mobility in healthy community-dwelling seniors: a meta-analytical review. Sports Med. 2016;46:1293-1309. doi: 10.1007/s40279-016-0485-1

17. Smith ML et al. Delivery of fall prevention interventions for at-risk older adults in rural areas: findings from a national dissemination. Int $\mathbf{J}$ of Env Res and Pub Health. 2018; 15:2798. doi: 10.3390/ijerph15122798

18. Scales K, Zimmerman S, Miller SJ. Evidence-based nonpharmacological practices to address behavioral and psychological symptoms of dementia. The Gerontologist. 2018;58:S88-S102. doi: 10.1093/geront/gnx167

19. Testad I, Corbett A, Aarsland D, Lexow KO, Fossey J, Woods B, Ballard C. The value of personalized psychosocial interventions to address behavioral and psychological symptoms in people with dementia living in care home settings: a systematic review. Int psychogeriatrics. 2014;26:1083-1098. doi: 10.1017/ S104161021400013
20. Spector A, Thorgrimsen L, Woods B, Royan L, Davies S, Butterworth M, Orrell M. Efficacy of an evidence-based cognitive stimulation therapy programme for people with dementia: randomised controlled trial. The British Journal of Psychiatry. 2003; 183:248-254. doi: 10.1192/bjp.183.3.248

21. Cheung G, Peri K. Challenges to dementia care during COVID-19: Innovations in remote delivery of group Cognitive Stimulation Therapy. 2020:1-3. doi: 10.1080/13607863.2020.1789945

22. Pitkala KH et al. Effects of psychosocial group rehabilitation on health, use of health care services, and mortality of older persons suffering from loneliness: a randomized, controlled trial. J of Gero Series A: Bio Sciences and Med Sciences. 2009;64: 792800. doi: 10.1093/gerona/glp011

23. Jansson AH, Savikko NM, Pitkälä KH. Training professionals to implement a group model for alleviating loneliness among older people-10-year follow-up study. Educ Gero. 2018;44:119-127. doi: 10.1080/03601277.2017.1420005

24. Zubatsky M, Berg_Weger M, Morley JE. Using Telehealth Groups to Combat Loneliness in Older Adults through COVID-19. J of the Am Ger Soc. 2020;68:16781679. doi: 10.1111 /jgs.16553

25. Bokolo A. Exploring the adoption of telemedicine and virtual software for care of outpatients during and after COVID-19 pandemic. Irish J of Med Sci. (1971-) 2020:1-10. doi: 10.1007/s11845-020-02299-z 